\title{
Computational Fluid Dynamics Modeling of a Supersonic Nozzle and Integration into a Variable Cycle Engine Model
}

\author{
Joseph W. Connolly*, David Friedlander \\ and \\ George Kopasakis; \\ NASA Glenn Research Center, Cleveland, OH 44135, USA
}

\begin{abstract}
This paper covers the development of an integrated nonlinear dynamic simulation for a variable cycle turbofan engine and nozzle that can be integrated with an overall vehicle Aero-Propulso-Servo-Elastic (APSE) model. A previously developed variable cycle turbofan engine model is used for this study and is enhanced here to include variable guide vanes allowing for operation across the supersonic flight regime. The primary focus of this study is to improve the fidelity of the model's thrust response by replacing the simple choked flow equation convergent-divergent nozzle model with a MacCormack method based quasi-1D model. The dynamic response of the nozzle model using the MacCormack method is verified by comparing it against a model of the nozzle using the conservation element/solution element method. A methodology is also presented for the integration of the MacCormack nozzle model with the variable cycle engine.
\end{abstract}

\section{Nomenclature}

$\begin{array}{llll}A & \text { Cross-sectional area } & \text { VGV } & \text { Variable guide vane } \\ \text { APSE } & \text { Aero-propulso-servo-elasticity } & \dot{W} & \text { Mass flow } \\ \text { ASE } & \text { Aero-servo-elasticity } & a & \text { Speed of sound } \\ \text { CESE } & \text { Conservation element/solution element } & c_{v} & \text { Specific heat at constant volume } \\ \text { CD } & \text { Convergent divergent nozzle } & e & \text { Internal energy } \\ \text { CFD } & \text { Computational fluid dynamics } & t & \text { Time } \\ \text { CFL } & \text { Courant-Friedrichs-Lewy stability condition } & u & \text { Velocity } \\ F & \text { Computational fluid dynamics flux vector } & x & \text { Length } \\ \text { FUN3D } & \text { Fully unstructured Navier-Stokes in } & \gamma & \text { Ratio of specific heat } \\ & \text { three dimensions } & \rho & \text { Density } \\ J & \text { Source term vector } & \text { Subscripts } \\ L & \text { Length } & N & \text { Total number of grid points } \\ \text { NPSS } & \text { Numeric propulsion system simulation } & b c & \text { Boundary condition } \\ M & \text { Mach number } & i & \text { Station location } \\ M F P & \text { Mass flow parameter } & s & \text { Static flow condition } \\ P & \text { Pressure } & t & \text { Total flow condition } \\ R & \text { Gas constant } & \text { Superscripts } \\ T & \text { Temperature } & \prime & \text { Non-dimensional variable } \\ U & \text { Solution vector } & * & \text { Throat location } \\ V & \text { Volume } & & \end{array}$

*Aerospace Engineer, Intelligent Control and Autonomy Branch, 21000 Brookpark Rd., 77-1, AIAA Senior Member.

${ }^{\dagger}$ Aerospace Engineer, Inlets and Nozzles Branch, 21000 Brookpark Rd., 5-12, AIAA Member.

${ }^{\ddagger}$ Senior Controls Engineer, Intelligent Control and Autonomy Branch, 21000 Brookpark Rd., 77-1, AIAA Member. 


\section{Introduction}

$\mathrm{T}$ HE NASA Fundamental Aeronautics Program, High Speed Project, aims to overcome the obstacles associated with supersonic commercial flight. This project aims to develop the technologies to allow for practical supersonic overland commercial transport at a Mach number slightly less than two. The proposed vehicles are long, slim body aircraft with the potential for pronounced structural vibrations known as aeroservo-elastic (ASE) modes. ${ }^{1,2}$ When coupled with propulsion system dynamics, the modes are known as aero-propulso-servo-elasticity (APSE). The APSE considerations can lead to design challenges pertaining to aircraft performance such as aircraft ride quality and stability. Furthermore, other disturbances upstream of the inlet generated by atmospheric wind gusts may also affect ride quality and stability. To study the relevant dynamics of the propulsion component of APSE, a nonlinear variable cycle engine (VCE) model was developed ${ }^{3}$ and is improved upon by incorporating a computational fluid dynamics (CFD) model of the convergent-divergent $(\mathrm{CD})$ nozzle.

CFD models of a supersonic CD nozzle are being developed and integrated into a VCE model to capture relevant thrust dynamics. Accurate representation of the thrust dynamics is critical for this research effort, as these models will ultimately be integrated into an aero-elastic vehicle dynamic model to investigate the physical coupling of the integrated vehicle and closed loop control performance.

This paper investigates the CFD modeling of a CD nozzle for a supersonic commercial transport using the MacCormack method. Comparisons of the steady state and dynamic result of the MacCormack method are verified against a Conservation Element/Solution Element (CESE) CFD approach for capturing the key dynamics of the $\mathrm{CD}$ nozzle. Integration of the MacCormack nozzle model into the previously developed VCE model is done to improve upon the simple choked flow equation that was previously used to model the nozzle. This higher fidelity modeling of the exit flow of the engine will allow for accurate capturing of the thrust dynamics required for the APSE task. The modeling approach used for the VCE model has previously been developed ${ }^{4,5}$ and verified. ${ }^{3}$ The work presented here for the VCE, however; expands upon the previous single design point to other key portions within the supersonic operating envelope through the implementation of variable stator vanes and exit nozzle areas. The work presented in this paper is only a portion of the broader dynamic modeling of the propulsion system for the needs of APSE. ${ }^{6}$

The overall paper objectives are as follows:

- Provide an overview of the supersonic CD nozzle model, with a focus on integration of the nozzle with the VCE propulsion system.

- Use of the CESE method for comparing dynamic modeling accuracy of the CD nozzle model.

- Illustrate the key features of the propulsion system model required to accurately capture the thrust dynamics.

The paper is organized as follows. First, a modeling overview that includes descriptions of the overall APSE goals, the CD nozzle, and the VCE model with a focus on variable guide vanes and nozzle integration. Second, a results section that will focus on the dynamic accuracy of the nozzle model and its integration with the VCE model for accurate thrust estimation. Third, the paper will outline the next steps required to complete the overall dynamic propulsion system, primarily the modeling of the external compression inlet, and its integration into the overall elastic vehicle model being developed in NASA Langley's fully unstructured Navier-Stokes in three dimensions (FUN3D) code. Finally, some concluding remarks will be provided on the performance of the integrated VCE-nozzle model.

\section{A. Supersonic APSE Overview}

\section{Modeling Overview}

The overall APSE simulation block diagram can be seen in Fig. 1. The propulsion system of the overall model, highlighted in Fig. 1, is comprised of the inlet, VCE, CD nozzle, and propulsion controllers. ASE vehicle impacts are neglected in this study, but are illustrated by dashed lines in Fig. 1 to provide an overview of how the propulsion system fits into the larger APSE simulation architecture. A mixed compression inlet was used in previous studies. ${ }^{5}$ However, the current project design is for an external compression inlet and will be modeled in this study using simple steady state performance calculations. All of the other propulsion elements are modeled using unsteady conservation equations, described in the following sections. This allows for the investigation of VCE control performance and thrust oscillations. To run the simulation, free stream 
static conditions of pressure, temperature, and Mach number are applied to obtain an inlet exit total pressure and temperature, which are then used as the engine face input conditions. The engine then uses the input conditions, its component performance maps, and a modified version of the Euler conservation equations to calculate the engine output provided to the CD nozzle interfaces. The version of the VCE used for the current study contains two CD nozzles, one for the main flow through the core and primary bypass called the primary duct nozzle, and a second for the variable cycle duct exit called the VCE nozzle. Both the primary duct and VCE duct nozzles are modeled using the MacCormack method.

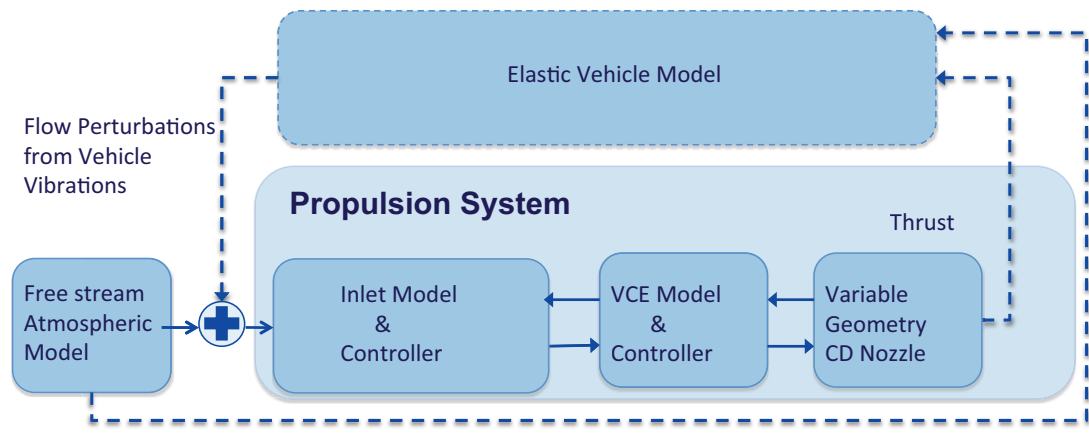

As discussed, an overall goal of the ASE project is to integrate a high fidelity vehicle and propulsion system model to investigate the dynamic performance coupled with the elastic vehicle. The work presented here is a step along that path, providing improved dynamic thrust estimation. Previous work in the area of APSE has focused on ei-

Figure 1. Block diagram of overall APSE system model with propulsion system highlighted. vehicle or the propulsion system, but rarely are both systems modeled with the same level of fidelity. In addition, most of the previous research has only considered a hypersonic vehicle where the vehicle itself provides compression of the flow feeding into the propulsion system. ${ }^{7-9}$ This leaves the speed regime of supersonics and the use of turbo-machinery as the propulsion system as an open field of research.

\section{B. Nozzle Model}

A CD nozzle was modeled to investigate the dynamic thrust behavior caused by upstream flow perturbations. As previously mentioned, the VCE propulsion system has two nozzles, one for the exit of the core and bypass duct called the primary duct nozzle and one for the variable cycle dict exit called the VCE duct nozzle. Both nozzles are modeled exactly the same with only geometric differences. The primary nozzle chosen for the supersonic transport includes a variable geometry CD internal duct and a conical plug or center body. The CFD approach for this study is geared towards having as simple of an approach as possible while still capturing the relevant thrust dynamics, thus a quasi-1D method is used. Thus, the approach described here is only suitable to model the internal duct portion of the two nozzles and neglects any effects of the external plug. This approach is justified by preliminary studies using a 2D CESE code that showed the external portion of the nozzle only contributed a few percent to the overall thrust. In addition, the ultimate goal of this work is to have a propulsion system model that will be integrated into NASA Langley's FUN3D ${ }^{10}$ code. This is a widely used 3D CFD code, where the propulsion system presented here will provide the dynamics of the internal duct and FUN3D will handle the more complex external flows.

Both a CESE and MacCormack quasi-1D method are used for modeling the internal duct of each nozzle, given the following Euler equations for mass, momentum, and energy conservation respectively:

$$
\begin{array}{r}
\frac{\partial(\rho A)}{\partial t}+\rho A \frac{\partial u}{\partial x}+\rho u \frac{\partial A}{\partial x}+u A \frac{\partial \rho}{\partial x}=0 \\
\rho \frac{\partial u}{\partial t}+\rho u \frac{\partial u}{\partial x}=-R\left[\rho \frac{\partial T}{\partial x}+T \frac{\partial \rho}{\partial x}\right] \\
\rho c_{v} \frac{\partial T}{\partial t}+\rho u c_{v} \frac{\partial T}{\partial x}=-\rho R T\left[\frac{\partial u}{\partial x}+u \frac{\partial \ln (A)}{\partial x}\right]
\end{array}
$$

The above equations Eq. 1 - 3 are perfectly fine to implement into a numerical scheme. However, for this study a non-dimensional form of the equations will be used. This is more style than substance, but can help to visualize changes in parameters without having large scaling issues for visualization. The dimensionless terms chosen are described below and denoted with a prime, ': 


$$
\begin{array}{r}
T^{\prime}=\frac{T}{T_{t}} \\
\rho^{\prime}=\frac{\rho}{\rho_{t}} \\
x^{\prime}=\frac{x}{L} a_{t}=\sqrt{\gamma R T_{t}} \\
u^{\prime}=\frac{u}{a_{t}} \\
t^{\prime}=\frac{t}{L / a_{t}} \\
A^{\prime}=\frac{A}{A^{*}}
\end{array}
$$

The conservative form of the governing equations was chosen for implementation. There is no mathematical difference from the non-conservative form, however the use of the conservative form can provide improved numerical results when the flow has discontinuities, such as the case of internal shocks in the nozzle. The conservative form can be written in a compact vector form as:

$$
\frac{\partial \mathbf{U}}{\partial t^{\prime}}=\frac{\partial \mathbf{F}}{\partial x^{\prime}}+\mathbf{J}
$$

where the components of the solution vector $(\mathbf{U})$, fluxes $(\mathbf{F})$, and source $(\mathbf{J})$ are:

$$
\begin{array}{r}
U_{1}=\rho^{\prime} A^{\prime}, U_{2}=\rho^{\prime} A^{\prime} u^{\prime}, U_{3}=\rho^{\prime} A^{\prime}\left[\frac{e^{\prime}}{\gamma-1}+\frac{\gamma}{2} u^{\prime 2}\right] \\
F_{1}=\rho^{\prime} A^{\prime} u^{\prime}, F_{2}=\rho^{\prime} A^{\prime} u^{\prime 2}+\frac{1}{\gamma} p^{\prime} A^{\prime}, F_{3}=\rho^{\prime} u^{\prime} A^{\prime}\left[\frac{e^{\prime}}{\gamma-1}+\frac{\gamma}{2} u^{\prime 2}\right]+p^{\prime} A^{\prime} u^{\prime} \\
J_{1}=0, J_{2}=\frac{1}{\gamma} p^{\prime} \frac{\partial A^{\prime}}{\partial x^{\prime}}, J_{3}=0
\end{array}
$$

The two modeling approaches allow for comparison and simple verification purposes. For the purposes of this paper the MacCormack method will be used as the primary modeling approach, meaning it will be the method that is integrated with the VCE model. The CESE method is an independent code used in this study for comparisons with the MacCormack method to improve the level of confidence of the nozzle model. The MacCormack method was chosen for integration with the VCE model for its simple finite difference approach. This approach more closely resembles the modeling method utilized for the engine. The following subsections will provide more details of the two approaches.

\section{MacCormack Method}

The MacCormack method is an explicit finite difference scheme that is very popular in CFD. This scheme allows for the accuracy of a Lax-Wendroff scheme, while reducing the overall complexity. The MacCormack method is multi-level, meaning that there is a multistep process to reach the solution. These steps are traditionally called predictor and corrector, where an averaging can then be performed. The averaging at each time step is what allows for the higher accuracy while using a relatively simple finite difference scheme. A step-by-step procedure of the MacCormack method can be found in Anderson. ${ }^{11}$

In the subsonic portion of the nozzle, one of the boundary condition variables is required to float, since information under this condition traverse both upstream and downstream. The choice was to allow the velocity to float to be consistent with the method outlined in Anderson. ${ }^{11}$ The boundary conditions are then defined in terms of the solution vector, where the density and temperature at the nozzle entrance are fixed:

$$
\begin{array}{r}
U_{1, i=1}=\rho_{b c} A_{1} \\
U_{2, i=1}=2 U_{2, i=2}-U_{2, i=3} \\
U_{3, i=1}=U_{1}\left(\frac{T_{b c}}{\gamma-1}+\frac{\gamma}{2} u^{2}\right)
\end{array}
$$


The boundary conditions at the nozzle exit are extrapolated using a simple linear relation, since the flow is assumed to have no internal normal shocks, and thus be supersonic:

$$
\begin{aligned}
& U_{1, N}=2 U_{1, N-1}-U_{1, N-2} \\
& U_{2, N}=2 U_{2, N-1}-U_{2, N-2} \\
& U_{3, N}=2 U_{3, N-1}-U_{3, N-2}
\end{aligned}
$$

\section{Computational Element / Solution Element Method}

The CESE method is a second-order accurate method that has been applied to a wide variety of CFD problems and has been proven to be a simple, robust, and efficient tool for dynamic simulations and aeroacoustic computations. A guiding principal of the CESE formulation is that space and time are unified and time is simply treated as an additional dimension to the problem. In addition, the enforcement of flux conservation in space and time at an interface is an integral part of the solution procedure. The method also uses a staggered mesh. The CESE method applied here for the modeling of a quasi-1D CD nozzle, expands upon the work by Chang for the modeling of a shock tube. ${ }^{12}$ The approach here follows Changs developing of the method applied to the Euler equations with the primary difference being that the equations above are used for a CD nozzle instead of a constant area shock tube as presented by Chang. This leads to the governing equations in the previous work needing to be modified to account for the source term that exists due to the area variation. Otherwise the exact same procedure is followed.

The boundary conditions for the CESE method are applied as outlined above in the MacCormack method, by simulating a subsonic entrance to the nozzle and supersonic exit. A slight variation to the application of the boundary conditions is that the interpolation and extrapolation need to account for the staggered grid.

\section{Engine Model}

The current engine design concept for the supersonic propulsion system is a variable cycle engine ( $\mathrm{VCE})$, which is an extension of a turbofan engine. The concept behind a VCE is to optimize the cycle throughout the flight regime by trimming the flow through the VCE fan stream so that the overall engine flow demand matches that of the inlet capture air. This is done in order to maximize pressure recovery and minimize flow spillage, which adds to drag.

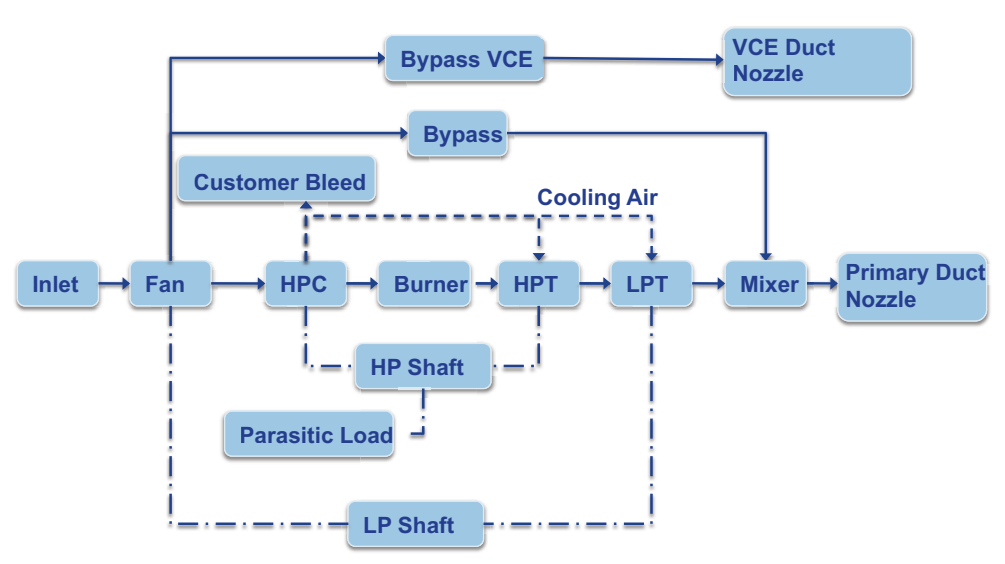

Figure 2. Engine design concept for a variable cycle engine.

The VCE fan stream is also utilized for noise abatement for supersonic overland flight.

The flow path of the VCE is split into three gas paths as illustrated in Fig. 2. The primary gas path is nearly identical to a turbojet and goes through the core of the engine. The secondary gas path is similar to a typical turbofan engine in that a large amount of the flow is bypassed around the core of the engine. In the VCE version covered in this paper, the main bypass flow is mixed with the core flow downstream of the turbine and exhausted out the common primary duct nozzle. The more advanced concept part of this engine design is a third gas path that exhausts through the VCE duct nozzle. The fan in Fig. 2 is split, such that the flow going to the bypass and core is one component model called fan, and the fan tip region feeding the third flow path is modeled by a component call VCE fan. The third flow path provides a lower exit velocity exiting the VCE duct nozzle that could be used as a noise shield for the flow exiting the primary duct nozzle. While Fig. 2 shows all of the major engine components that have their own conservation equations, in between each component is also an associated connecting duct that is not shown in the figure for simplicity. Each of these ducts are also modeled using the governing conservation equations. ${ }^{4}$ In addition, a preliminary fan speed controller is included that was previously developed. ${ }^{13}$ 
The initial operating conditions and component geometry information, as well as the component performance maps required to implement the dynamic VCE model is obtained from a design concept of a supersonic VCE implemented in the Numerical Propulsion System Simulation (NPSS). ${ }^{14}$ A cruise operating point was chosen at 15,240 $\mathrm{m}(50,000 \mathrm{ft})$ and a Mach number of 1.6. A choked nozzle flow equation has previously been used for the exit boundary condition. The VCE model uses a single lumped volume for each of the major components such as compressor, combustor, turbine, afterburner, and nozzle. The modeling approach is outlined in previous works. ${ }^{3,4,15}$ Each of the fluid flow components is modeled using a set of derived conservation equations modified from the standard Euler form and written for continuity, momentum, and energy. These equations are integrated numerically using a time marching scheme and the Seldner differencing technique of the spacial terms. ${ }^{3}$

In typical subsonic commercial aircraft, CFD models of turbofan engines have not been necessary for control design due to the operating conditions and expected lower frequency perturbations. For such models in this operating regime, only the turbofan shaft inertias have been required for closed-loop controls development. However, for new commercial supersonic transports the assumptions of just being able to develop propulsion system controls around shaft dynamics may no longer be valid.

\section{Variable Cycle Engine Variable Guide Vane Modeling for Expanded Flight Envelope}

The primary update of the dynamic VCE model for the work presented here from previous studies is the inclusion of variable guide vanes (VGV) that allow for the engine to operate across its full supersonic operating envelope. Previous models where designed about a single operating point. However, numerical instabilities could be encountered when attempting to operate these models at other points throughout the supersonic operating envelope. The VGV model is developed and integrated with the compressor components, which include the high pressure compressor, fan, and VCE fan. This allows for variable geometries necessary to operate the VCE within its operating range of altitude, Mach, and power, while forcing the compressors (via control schedules) to operate on a desired operating line of the compressor performance maps.

The VGV model consists of performance characteristics with sets of maps at different VGV angles, which are interpolated. An example of the VCE fan performance map and its variation with VGV angle is illustrated in Fig. 3. The solid black lines represent corrected speed and the color map represents the operating efficiency. For this dynamic model, the effective cross sectional area is calculated as a function VGV angle. The maps supplied by the NPSS model include the overall incidence angle. The incidence angle, however, is taken out in this model and the reference angle with no VGV turning is referred to as the nominal angle. This nominal angle corresponds to the initialized compressive component cross sectional area. For the VGV model, the mass flow parameter $\left(M_{F P}\right)$ is utilized and the compressor effective flow area is calculated as a function of the $M_{F P}$. Additionally, by taking into account the cosine of the VGV angle as

$$
\begin{array}{r}
A=\frac{\dot{W} \sqrt{T_{t}}}{M_{F P} P_{t} \cos (\alpha)} \\
M_{F P}=M \sqrt{\frac{\gamma g}{R}}\left(1+\frac{\gamma+1}{2} M^{2}\right)^{\frac{\gamma+1}{2(1-\gamma)}}
\end{array}
$$

where $A, M_{F P}, \dot{W}, P_{t}, T_{t}, \cos (\alpha)$, and $M$ are the component cross section area, the mass flow parameter, the component total pressure and temperature, the cosine of the VGV angle, and the component Mach number. The angle, $\alpha$, is the VGV turning angle. 

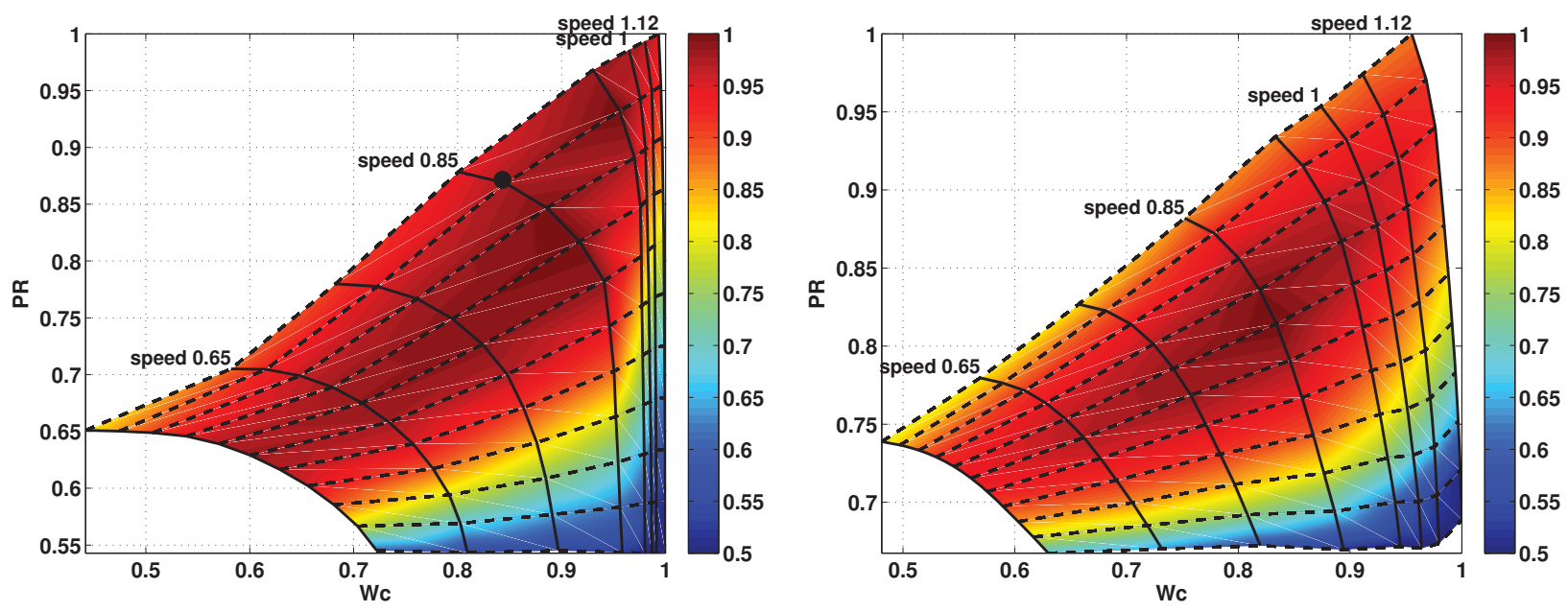

Figure 3. The VCE fan compressor performance maps at a low VGV angle (left) and a high VGV angle (right), where the color map corresponds to efficiency.

\section{Engine and Nozzle Integration}

The integrated nonlinear propulsion system used in this study includes the VCE and CD nozzle models. This allows for the development of the modeling approach, control concepts, and interfaces for the overall propulsion system integration and APSE interfaces. Ultimately, an external compression inlet needs to be developed and integrated with the VCE to have a more accurate picture of how atmospheric disturbances will impact thrust for the current supersonic design concepts. This effort is currently ongoing. ${ }^{6}$

The integration approach is to replace the previous choked flow equation of the engine model exit boundary condition with the MacCormack method CFD CD nozzle described in the preceding sections. The choked flow boundary condition is replaced with a very small duct volume that can be added to the engine simulation. This duct volume is modeled utilizing volume dynamics, similar to the approach applied in modeling the ducts in the VCE, where the conservation equations become Eqs. (22) to (24).

$$
\begin{array}{r}
\frac{d}{d t}\left(\rho_{s}\right)=\frac{1}{V}\left(\dot{m}^{v c e_{e x i t}}-\dot{m}^{n o z z l e}\right) \\
\frac{d}{d t}(\dot{m})=\frac{A}{x}\left(P_{s}^{v c e_{d u c t}}-P_{s}^{v c e_{e x i t}}\right) \\
\frac{d}{d t}\left(\rho_{s} T_{t}\right)=\frac{\gamma}{V}\left(T_{t}^{v c e_{d u c t}} \dot{m}^{v c e_{e x i t}}-T_{t}^{v c e_{e x i t}} \dot{m}^{\text {nozzle }}\right)
\end{array}
$$

This duct volume serves as the VCE-nozzle interface used to calculate the states and the static density and temperature required for the nozzle model. The MacCormack nozzle then provides the mass flow rate that was previously supplied by the choked flow equation. The superscripts used in the above equations indicate the location of the variable relative to the VCE-nozzle interface. These equations are then tied together using the state equation. The nozzle model then provides the dynamic estimation of gross thrust.

\section{E. Engine Fan Speed Controller}

The engine control design used in this study has aspects of classical loop shaping ${ }^{16}$ design and quantitative feedback theory. ${ }^{17}$ The engine controller for the fan speed was first designed based on linear models, ${ }^{13}$ and later implemented in the nonlinear VCE model. The fan speed is controlled using the engine fuel injector that has a bandwidth of $6 \mathrm{~Hz}$. In addition, the VCE engine design has variable nozzle and VGV that are set on schedules and not actively controlled. The nozzle geometry and VGV is currently scheduled based on the corrected core speed. 


\section{Results}

The purpose of these simulation results is to demonstrate the steady-state accuracy and dynamic performance of the CD nozzle model using the MacCormack method and the integrated propulsion system model with a fan speed controller. The results will be broken out into two sub-sections; one for the stand-alone nozzle component verification and the other for the nozzle integrated with the VCE. Each of the sub-sections will first discuss the steady state accuracy and then focus on the dynamic performance. The VCE-nozzle integration sub-section will focus on the thrust dynamics.

\section{A. Nozzle}

This sub-section will investigate the steady state and dynamic results for the primary duct nozzle. The VCE duct nozzle model is also included for the final integration with the VCE, however, for brevity only the primary duct results are presented here. Comparison with the CESE method are provided and further studies are done with the MacCormack method, since this is the method utilized for the final nozzle model that is integrated with the VCE model. The results presented here are for a cruise condition of Mach 1.6 and $50,000 \mathrm{ft}$.

\section{Steady State}

The first comparison of the MacCormack and CESE nozzle are simply the steady state results compared to the known analytical solution based on the area-Mach number relationship. ${ }^{11}$ Steady state results from these simulations for the primary duct are shown in Fig. 4. The comparison is done across the length of the nozzle for the nondimensional primitive variables of density, velocity, and temperature. The MacCormack method is shown as solid lines, the CESE method as dashed lines, and the analytical solution as "x's". It can be seen that the CESE solution is in excellent agreement with the MacCormack method and analytical solution. The only slight deviation shown is near the throat of the nozzle, located at an axial location of 0.55 .

A further study using the MacCormack method was conducted to determine the steady state accuracy

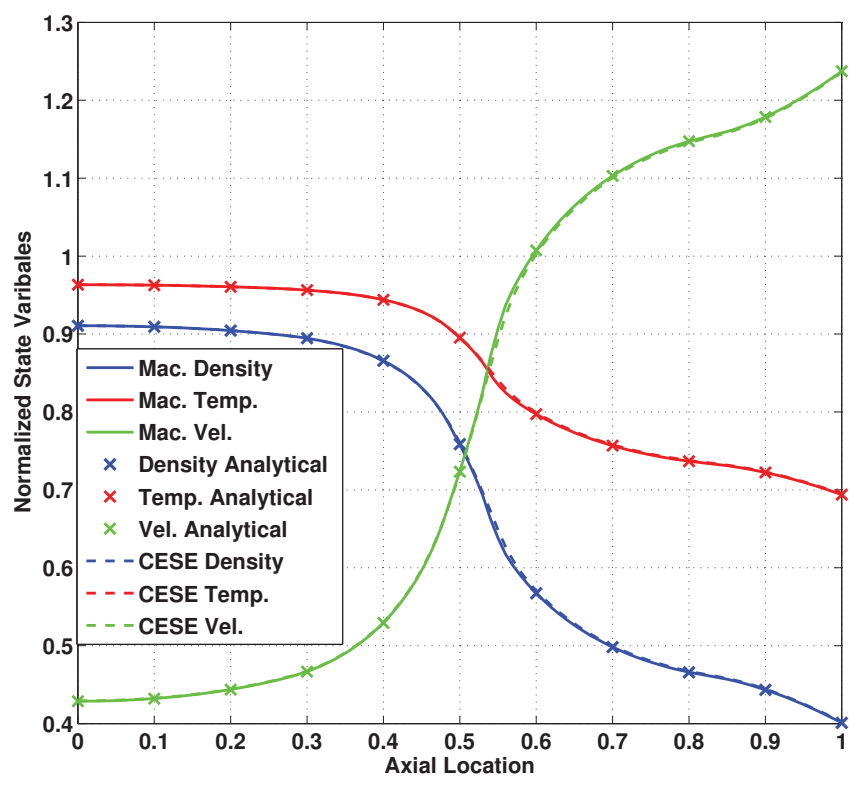

Figure 4. Nozzle steady state comparison for MacCormack, CESE, and analytical solution for normalized static density, temperature, and velocity.

with various grid sizes compared to

the analytical solution. The results are tabulated in Table. 1. The internal grid points, neglecting the boundary conditions, are used to calculate the mean percent error across the entire computational domain of the nozzle. The steady state results show that even with a very sparse grid of 21 points, the mean error is less than 1\%, and substantially decreases with larger grid sizes. Note, while the grid sizes where increased, the Courant-Friedrick-Lewy (CFL) stability condition was held constant at 0.95 , which essentially changes the time step of the simulation as well. 
Table 1. Nozzle steady state mean absolute percent error with various grid size using the MacCormack method.

\begin{tabular}{|l|l|l|l|}
\hline Primitive Variable & $21 \mathrm{pts}$ & $101 \mathrm{pts}$ & $201 \mathrm{pts}$ \\
\hline \hline Velocity & 0.10 & 0.0088 & 0.001 \\
\hline Temperature & 0.21 & 0.0026 & 0.00014 \\
\hline Density & 0.67 & 0.016 & 0.00289 \\
\hline
\end{tabular}

\section{Dynamic}

For the dynamic results, a grid study of the MacCormack method was conducted to determine the relative number of nodes required to get minimal changes in the magnitude and phase of a bode plot spanning the frequencies of interest. The highest expected frequency due to atmospheric turbulence at the desired cruise condition of less than Mach 2 is about 20Hz. However, the resulting aero-elastic disturbances due to the vehicle vibrations could get as high as $60 \mathrm{~Hz}$. This dictates that the frequency range of interest is extended to $600 \mathrm{~Hz}$, since dynamics up to this frequency can still effect the phase at $60 \mathrm{~Hz}$. Dynamic results for the primary duct using the MacCormack method were generated where various grid sizes are used to investigate impact on dynamic accuracy.

The grid study is done using a sinusoidal logarithmic sweep of $1 \%$ amplitude on the nozzle density entrance boundary condition, and then compared to the density at the nozzle exit. The resulting bode plot is shown in Fig. 5. The left of Fig.5 shows the full frequency range from 6 to $600 \mathrm{~Hz}$, and the right side is a close up at the higher frequency range. It is illustrated that even a very small number of nodes can provide reasonable accuracy at frequencies of less than $100 \mathrm{~Hz}$, but the sparse grids begin to show deviation beyond that frequency. From these results it was determined that 101 nodes or grid points would be sufficient for the frequency range of interest. This was determined since the variation from the case with 201 nodes at the $600 \mathrm{~Hz}$ range was less than $1 \mathrm{~dB}$ in magnitude and only a couple degrees in phase.
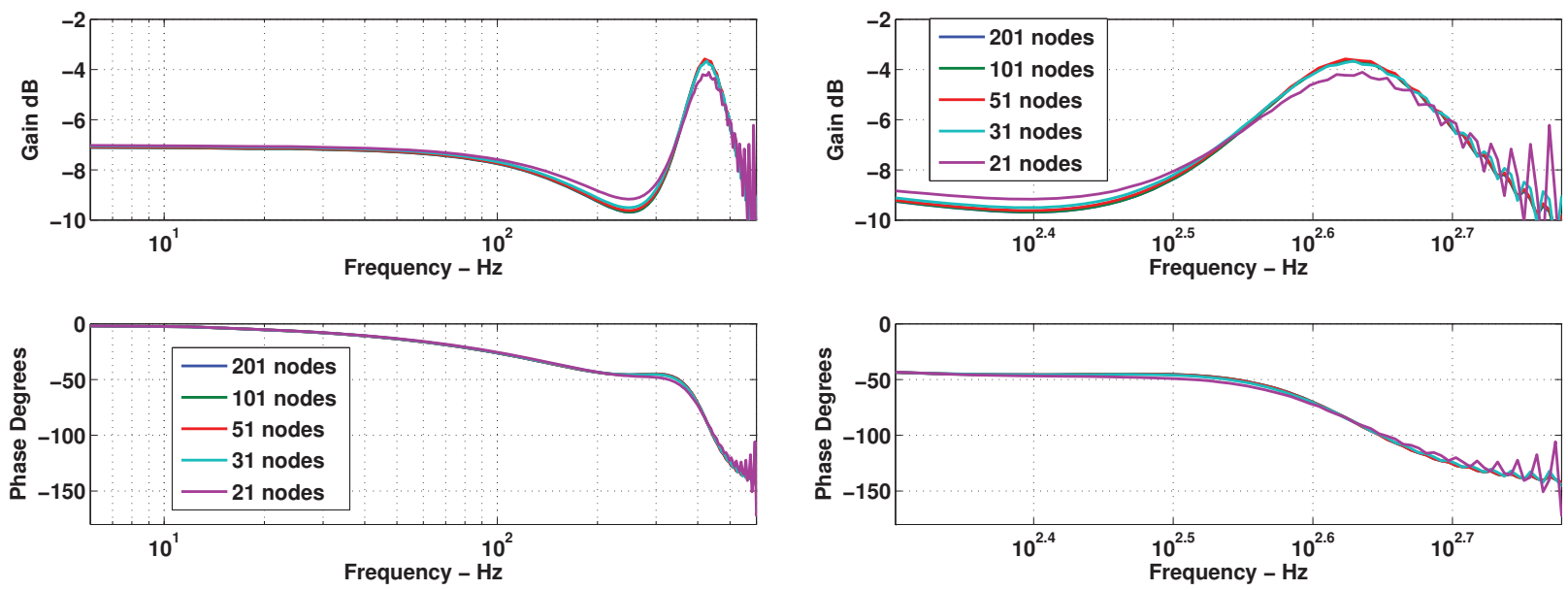

Figure 5. Nozzle exit density bode plot obtained from a logarithmic sinusoidal density wave applied at the nozzle entrance boundary condition for investigating the dynamic accuracy due to grid sensitivity.

Upon completion of the grid study, the MacCormack and CESE methods were compared at various constant frequencies. Similar to the bode plots in the grid study a $1 \%$ amplitude disturbance on the nozzle density entrance boundary condition is compared to the density at the nozzle exit, but now using a constant sine wave. The results of such a comparison at $1000 \mathrm{~Hz}$ are shown in Fig. 6. The top plot shows the normalized disturbance of density subtracted by the steady state value versus non-dimensional time units. The bottom plot shows the results of the density response at the nozzle exit for both the MacCormack (black) and CESE (red) methods. The results show that the two methods agree very well dynamically even 
at higher frequencies.
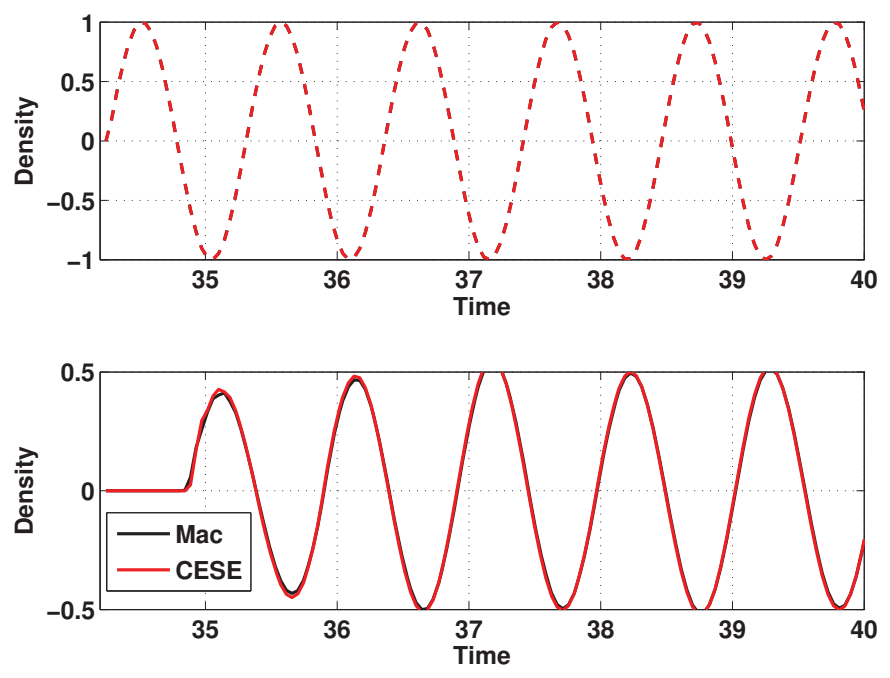

Figure 6. Nozzle entrance boundary condition disturbance of density using a constant sine wave at $1000 \mathrm{~Hz}$ normalized with the mean removed versus non-dimensional time units.

The constant sine wave disturbance described above is then repeated at various discrete frequencies spanning the frequency range of interest. The input to output response is then determined and used to generate a bode plot. This is done in order to compare the frequency response of the MacCormack method against the CESE method for discrete frequencies, with the results shown in Fig. 7. In the figure, the discrete results using the MacCormack method are plotted as blue crosses and the CESE method are shown as red circles. It can be seen that two methods agree very well, and provide a high level of confidence in the nozzle dynamics in the absence of experimental data. In addition to the discrete sine waves, a logarithmic sinusoidal sweep is applied using the MacCormack method to obtain the frequency response shown as a solid blue line. The sinusoidal logarithmic sweep is a more desirable method to evaluate the dynamic response as it takes much less computation time. It is assumed here that the dynamic response obtained by the discrete frequencies is more accurate than the sweep. The results shown in Fig. 7 illustrate that the sweep is able to accurately obtain the magnitude response, with only an error of about $0.5 \mathrm{~dB}$. The accuracy of the phase is not as good, particularly at the higher frequencies. However, even at the higher frequencies the difference between the sweep and constant sine waves is only about ten degrees. The most critical frequencies to accurately model are $60 \mathrm{~Hz}$ and below, so with an error of only ten degrees a decade above the frequency of interest this result shows that if a quick evaluation of the dynamics is required a sweep can be used with relatively good accuracy.
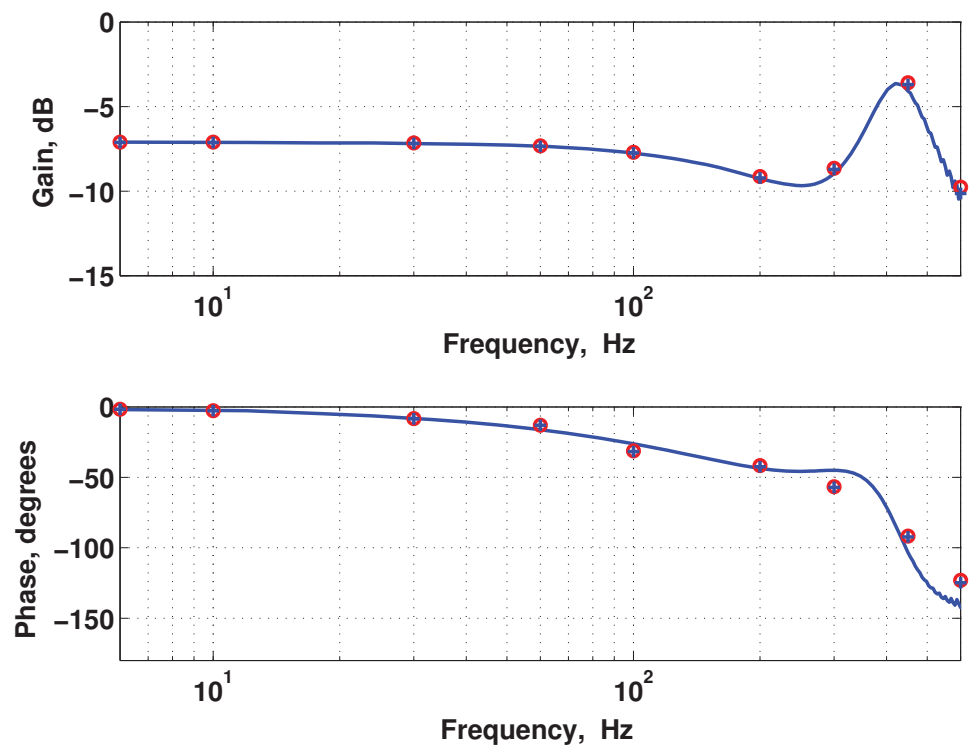

Figure 7. Bode plot of the Nozzle density input to output comparing a $1 \%$ amplitude constant sine wave using the MacCormack method (blue cross) to the CESE method (red circle). In addition, a logarithmic sinusoidal sweep is shown using the MacCormack method as the solid blue line. 


\section{B. Engine-Nozzle Integration}

The following results are for the VCE model integrated with the MacCormack method model of both the primary duct and variable cycle duct nozzles. The following section will outline the steady state comparison of the model compared to the initialization data from the NPSS model, the fan speed control response, and the gross thrust response. The results presented here are for a cruise condition of Mach 1.6, 50,000ft, and $100 \%$ power, unless otherwise stated.

\section{Steady State}

The VCE dynamic model is initialized by steady state data provided by the NPSS model of the VCE. The steady state percent difference between the NPSS model initialization data and the dynamic model of key engine components for mass flow, density, and temperature is illustrated in Table 2 . The results show that the absolute percent error is typically less than $2 \%$, however the compressive components have slightly larger errors. The errors here are most likely due to slight geometry differences between the NPSS and dynamic model, and differences in the interpolation schemes for the performance maps. In general the steady state results are expected to be reasonable from a controls perspective.

Table 2. Engine-Nozzle steady state absolute percent error.

\begin{tabular}{|l|l|l|l|}
\hline Engine Components & Mass Flow & Density & Temperature \\
\hline \hline VCE Fan & 2.65 & 4.34 & 1.43 \\
\hline Fan & 0.01 & 2.37 & 0.70 \\
\hline HPC & 2.05 & 2.61 & 4.35 \\
\hline Combustor & 0.49 & 0.51 & 0.69 \\
\hline HPT & 0.94 & 0.08 & 1.66 \\
\hline LPT & 0.94 & 1.09 & 0.99 \\
\hline
\end{tabular}

\section{Dynamic}

The following results will highlight the dynamic response of the integrated propulsion system, which includes the VCE engine and CD nozzles using the MacCormack method. The results in this section will again use the previously discussed cruise conditions at Mach 1.6. To begin, the VCE controlled response is investigated. The $\mathrm{VCE}$ has a simple fuel actuator with a bandwidth of $6 \mathrm{~Hz}$, and fuel flow is regulated to control fan rotational speed. A more detailed discussion of the controller can be found in previous papers covering the $\mathrm{VCE}$ engine development. ${ }^{5,13} \mathrm{~A}$ simple series of commanded fan speed steps up and down with a percent change of $1 \%$ from nominal are used to illustrate the controlled response and are shown in Fig. 8. The dis-

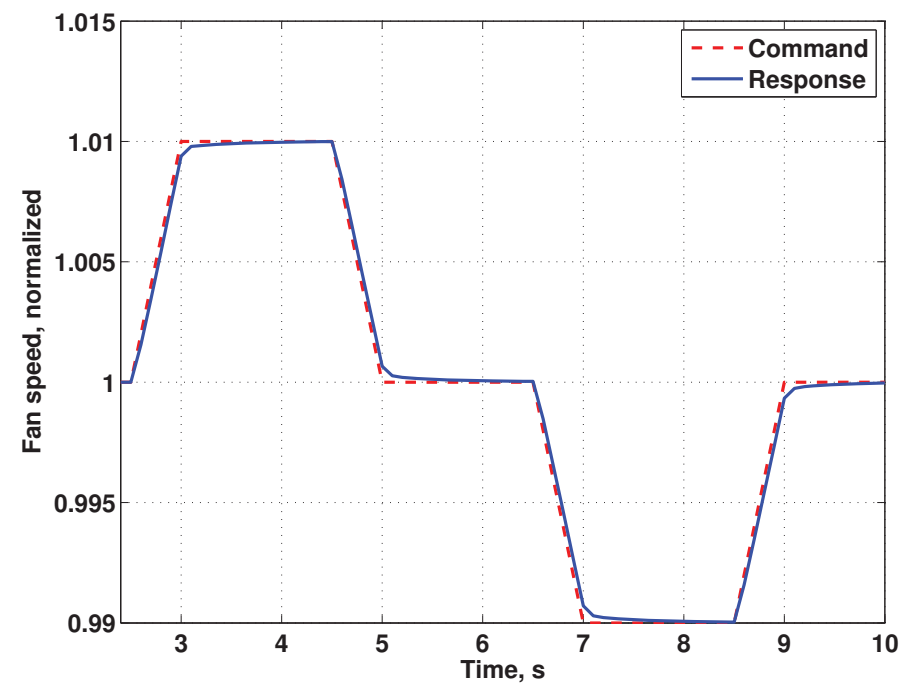

Figure 8. Engine-nozzle fan speed commanded steps and corresponding fan speed response. turbances in this section are applied after 2.5 seconds to allow for the startup transient to settle out. The dashed red line here is the commanded 
fan speed and the solid blue line is the response of the fan speed. This modest control demand is just used to illustrate that the propulsion system can operate about and maneuver around the cruise operating condition.

The primary enhancement to the VCE model from previously published work is the inclusion of the VGV for the compressive components that allow for operation about the supersonic flight regime. Operation across this flight regime is illustrated in Fig. 9. The plots shown here are again for a commanded fan speed up and down of $1 \%$ from nominal as was discussed above. The plots are of the resulting thrust response normalized by the nominal output thrust for the Mach 1.6 operating condition, and the fuel flow is regulated to a specified fan speed. The thrust responses are all very similar, with the only significant difference being the steady state thrust output of the engine as expected. In the top plot of Fig. 9, the gross thrust output is increased by about $44 \%$ to operate at a cruise Mach of 1.2 and a decreased altitude of 44,000 ft. The bottom plot shows the reduction of the thrust output in relation to the nominal cruise condition to about $84 \%$ when operating at Mach 2.0 and an increased altitude of 52,000ft.
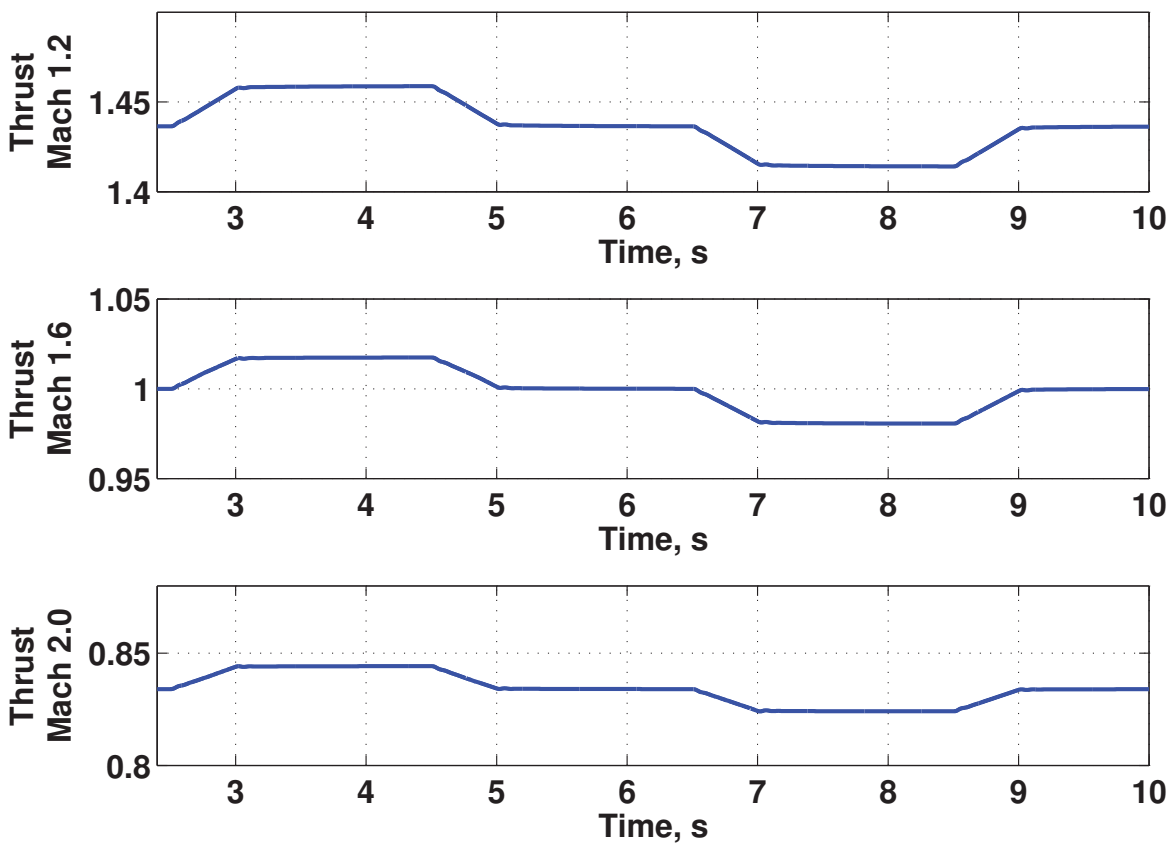

Figure 9. The VCE-nozzle simulated gross thrust response due to steps up and down in fuel flow at Mach 1.2 and altitude of 44,000 ft (top plot), Mach 1.6 and altitude of 50,000 ft (middle plot) and Mach 1.8 and altitude of $52,000 \mathrm{ft}$ (bottom plot).

The thrust response of the VCE integrated with the MacCormack method for both the VCE and primary duct nozzles is now compared with the previously used simple choked flow equation. To begin, the same commanded fan speed steps are used at the nominal cruise condition of Mach 1.6 and altitude of 50,000 $\mathrm{ft}$. This is done to illustrate the lower frequency thrust dynamics and is shown in Fig. 10. The overall steps up and down are shown in the left most plot where it can be seen that the responses are very similar. The propulsion system with the MacCormack method model of the nozzles has a slightly more significant response to the steps up and down in fan speed causing a small steady state offset when using the choked flow equation. The right plot of Fig. 10 is simply a close up and shows that the transient responses of the initial step up are very similar between the two propulsion system models.

The thrust response shown in Fig. 10 was not entirely unexpected as the flow in the nozzle is supersonic and slower dynamic responses should not show significant differences. However, at higher frequencies the nozzle model using the MacCormack method will be able to capture the relevant dynamics that the simple choked flow equation cannot. For illustrative purposes, a constant density sine wave perturbation is applied at the nozzle entrance at a frequency of $600 \mathrm{~Hz}$ (the highest frequency of interest) and $1 \%$ amplitude. This is used to compare the simple choked flow equation to the MacCormack method shown in Fig. 11. Here it 

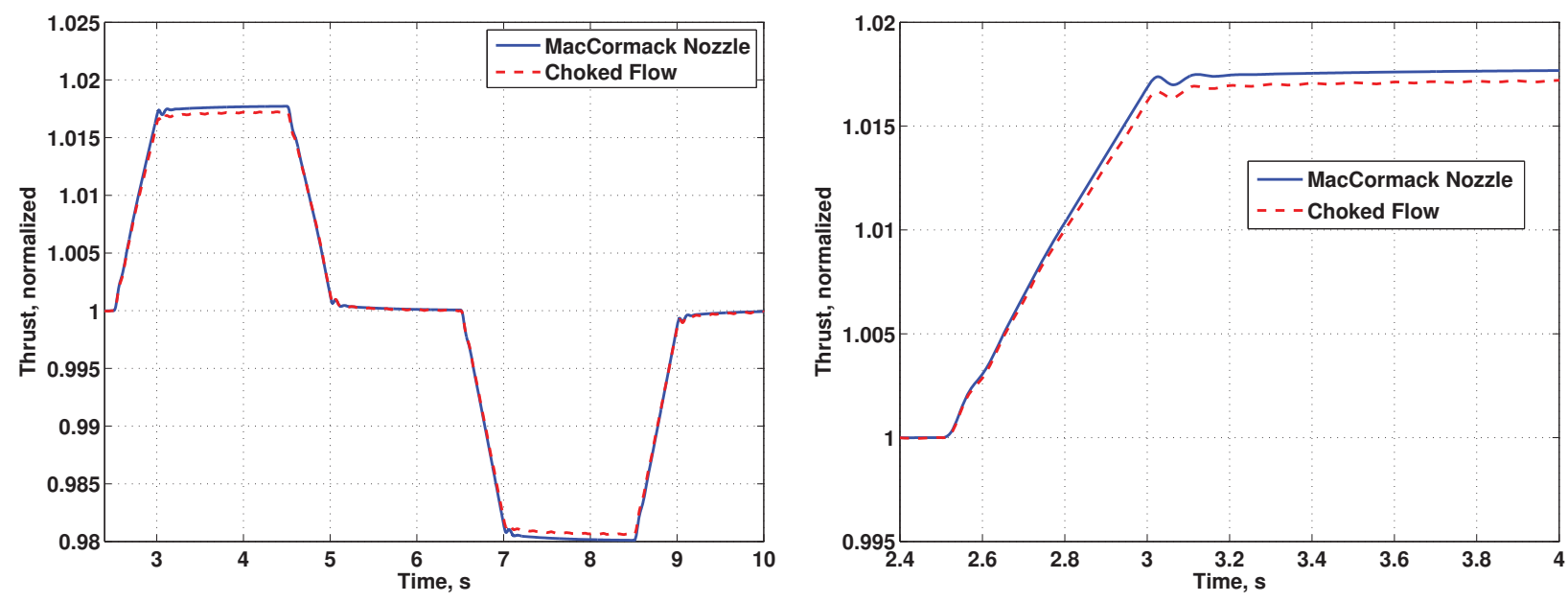

Figure 10. Propulsion system normalized thrust response to commanded fan speed steps up and down (left) and a close up of the first step up (right).

can be clearly seen that the perturbation using the choked flow equation flows directly through the nozzle, dashed red line, without any delay or added dynamic response. The MacCormack method does account for the time delay of the perturbation to flow through the nozzle before it impacts the thrust response and it captures some added dynamics of the nozzle. This leads to the two responses being out of phase, however the time scale is very small.

Finally, a pressure perturbation is applied at the entrance to the engine face using a constant sine wave of $60 \mathrm{~Hz}$ and an amplitude $1 \%$ of the nominal steady state pressure. The frequency of the perturbation was chosen as the highest expected aero-elastic disturbance frequency. Ultimately for the APSE studies, the perturbations of interest will be atmospheric wind gusts in the free stream. However for the present study, a simple pressure perturbation is applied at the engine entrance face due to the absence of an inlet dynamic model. Normally the external compression inlet should provide some dissipation of the free stream perturbation, so this could be thought of as more severe than applying a $1 \%$ pressure perturbation to the free stream conditions. This disturbance is illus-

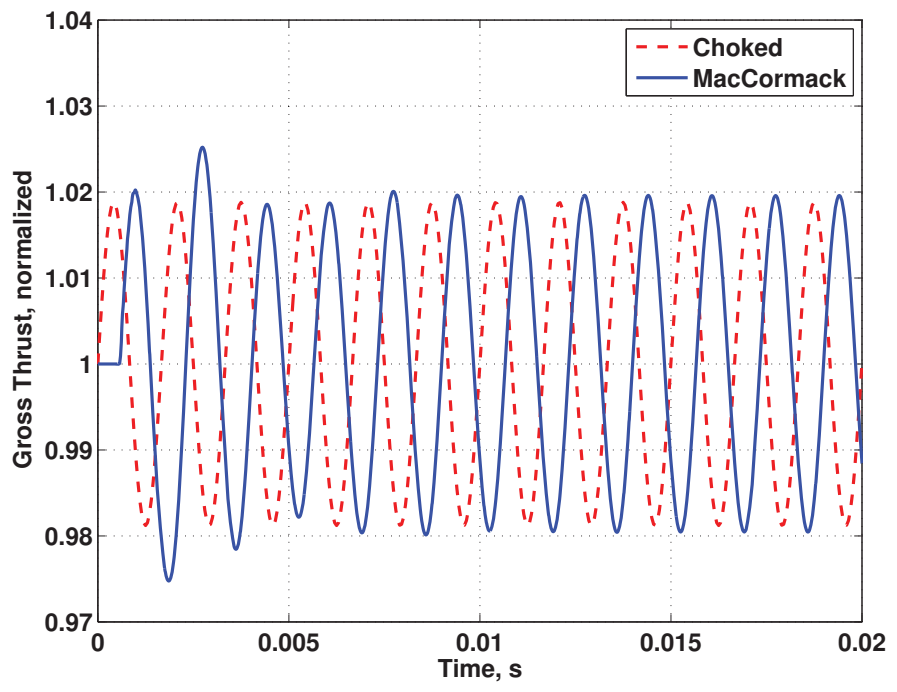

Figure 11. Nozzle thrust response due to $600 \mathrm{~Hz}$ constant density perturbation at the nozzle entrance, comparing the simple choked flow and MacCormack modeling approaches. trated in the top plot of Fig. 12

The bottom plot of Fig. 12 shows the resulting thrust responses of the propulsion system with using either the simple choked flow equation or MacCormack method model of the nozzles. The two response are different with the MacCormack method having a slightly smaller amplitude response to the initial perturbation, but the main take away is that the two response are very similar. While the MacCormack method has been shown to capture the relevant dynamics of the nozzle as shown in Fig. 11, when a perturbation is applied at 
the engine face the dynamics of the engine and the fuel flow controller are dominate. The dynamics of the nozzle at this given supersonic condition and geometric design causes the nozzle to not play a major role in the thrust dynamics of interest for APSE. An operational region where this could change for the proposed flexible supersonic vehicle is the case of a takeoff condition, where the vehicle will be at subsonic speeds and the dynamics of the flow through the nozzle could change.
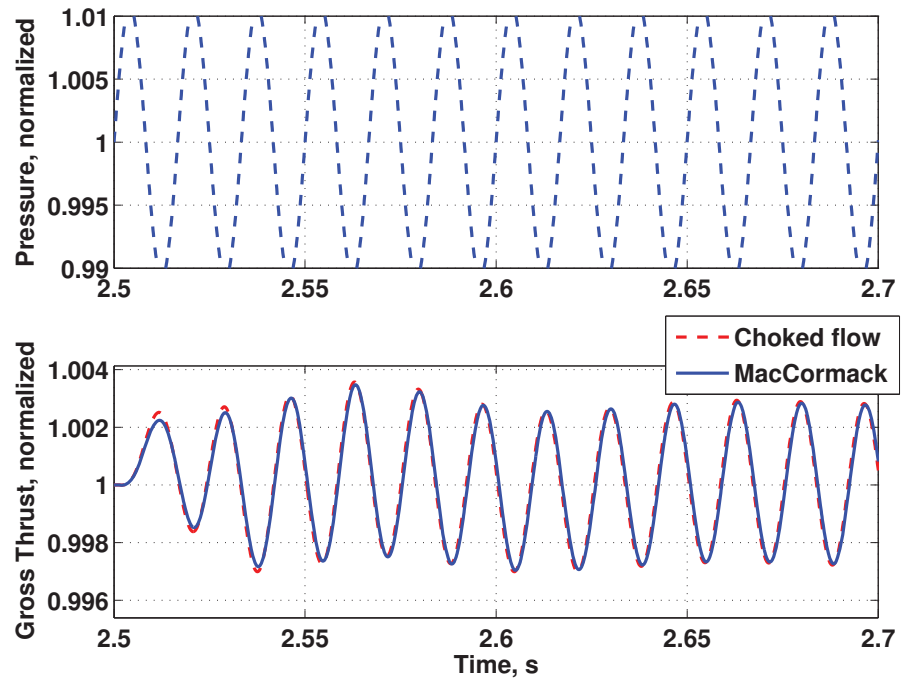

Figure 12. Nozzle thrust response to a constant $60 \mathrm{~Hz}$ sine wave pressure perturbation of $1 \%$ amplitude at the engine entrance face and the corresponding thrust response for both the VCE with the choked flow equation and MacCormack method nozzles. simulated by only utilizing the choked flow equation, this will reduce the complexity and execution time of the propulsion system simulation.

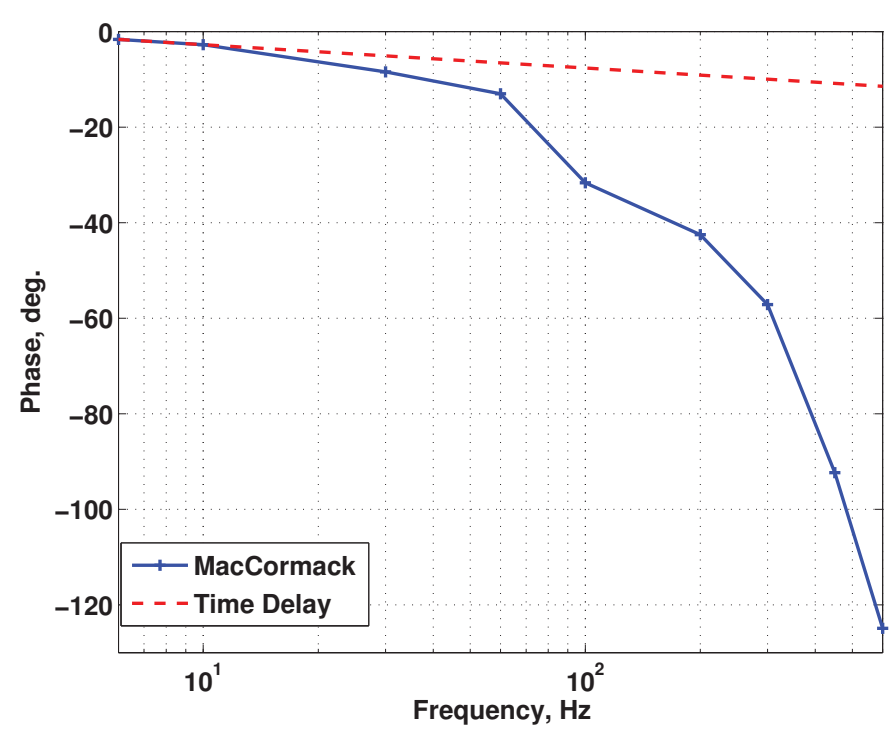

Figure 13. MacCormack method nozzle compared to a choked flow equation nozzle model with a flow propagation time delay using discrete frequency density perturbation sinusoids over the frequency range of interest to investigate phase change. model of the nozzle would be to include a time delay to approximate the flow propagation time across the nozzle length. Since the ASE coupling frequency can be as high as $60 \mathrm{~Hz}$, the dynamic accuracy of the phase at this frequency between the MacCormack nozzle simulation and the choked flow nozzle equation is critical for control design. To investigate the accuracy of the simple time delay compared to the MacCormack method, data from Fig. 7 is duplicated and a straight line approximation of the time delay phase shift in $\log$ scale is shown in Fig. 13. Figure 13 shows that this difference in phase at $60 \mathrm{~Hz}$ is only about 6 degrees, which would be acceptable as an error for closed-loop APSE dynamics for control stability. Obviously, if the nozzle dynamics can be

A simple way to increase the fidelity of the choked flow equation 


\section{Future Work}

To complete the overall propulsion system model, a nonlinear dynamic model of the external compression inlet is required to capture all of the relevant dynamics that could impact the thrust response. While it has been shown here that the nozzle may not play a major role in capturing the relevant dynamic response of thrust for the purposes of studies in APSE, it is expected that the inlet will play a significant role. This is primarily due to the inlet being the interface of the propulsion system to the free stream perturbations of interest in APSE. In addition, the inlet is a large volume where the velocity of the fluid is relatively slow compared to that of the nozzle. Moving forward, the MacCormack method for the nozzle will continue to be used in the final development of the overall propulsion system to get a full assessment of the thrust dynamics. The importance of the nozzle dynamic accuracy could play a role once the inlet is connected to the overall propulsion simulation or operation of the propulsion system at flight conditions other than those explored in this study. Upon completion of the inlet dynamic model and its integration with the VCE model, the overall propulsion system needs to be included in an overall simulation of the aero-elastic vehicle. Work is currently underway for incorporating the propulsion system models used in this study into NASA Langley's FUN3D code.

\section{Conclusion}

An integrated engine and nozzle propulsion system component model has been developed with a fan speed controller, where the engine components have previously been reported upon. The integrated propulsion system model presented in this work is suitable for incorporation into an overall supersonic vehicle aeropropulso-servo-elastic (APSE) model where accurate thrust dynamics are of primary concern. A convergentdivergent nozzle model has been developed for replacing the relatively simple choked flow equation model previously used in the engine model to improve the thrust dynamic response. The nozzle model is developed using the MacCormack method and its dynamic accuracy is compared against a nozzle model using a conservation element/solution element method. The dynamic comparison of the two approaches showed very good agreement. In addition to replacing the simple choked flow nozzle of the engine model, variable guide vanes for the compressive components were also included. This allowed for operation of the propulsion system across its supersonic flight regime, which was previously not possible. The engine-nozzle integrated propulsion system showed that at supersonic cruise when including the higher fidelity MacCormack nozzle models perturbations at the engine face do not have a significant impact on the overall thrust response compared to the simple choked flow model. For the desired cruise operating condition and this specific nozzle design, a simple choked flow model for the engine nozzle should be sufficient to capture the relevant thrust dynamics for APSE needs, as the dynamics of the other engine components and fan speed controller dominate the thrust response.

\section{Acknowledgements}

The authors would like to express their gratitude to the High Speed Project of the NASA Fundamental Aeronautics Program for supporting this research effort, Xiao-Yen Wang for advice on applying the Conservation Element and Solution Element method to develop the nozzle model, and finally John Muich for his assistance initial verification of results from the MacCormack method of the nozzle model.

\section{References}

\footnotetext{
${ }^{1}$ Cohen, P., Long-Davis, M., and Povinelli, L., "Fundamental Aeronautics Program - Supersonics Project," http://www.aeronautics.nasa.gov/fap/documents.html, 2006.

${ }^{2}$ Perry, B., Silva, W., Florance, J., Wieseman, C., Potozky, A., Sanetrik, M., Scott, R., Keller, D., Cole, S., and Coulson, D., "Plans and Status of Wind Tunnel Testing Employing an Aeroservoelastic Semispan Model," 48th AIAA/ASME/ASCE/AHS/ASC Structural Dynamics, and Materials Conference, No. AIAA-2007-1770, April 2007.

${ }^{3}$ Kopasakis, G., Connolly, J., Paxson, D., and Ma, P., "Volume Dynamics Propulsion System Modeling for Supersonics Vehicle Research," Journal of Turbomachinery, Vol. 132, No. 4, October 2010, pp. 8.

${ }^{4}$ Connolly, J., Kopasakis, G., and Lemon, K., "Turbofan Volume Dynamics Model for Investigations of Aero-PropulsoServo-Elastic Effects in a Supersonic Commercial Transport," 45th AIAA/ASME/SAE/ASEE Joint Propulsion Conference and Exhibit, No. AIAA-2009-4802, 2009.

${ }^{5}$ Connolly, J., Kopasakis, G., Paxson, D., Stueber, E., and Woolwine, K., "Nonlinear Dynamic Modeling and Controls Development for Supersonic Propulsion System Research," 47th AIAA/ASME/SAE/ASEE Joint Propulsion Conference and Exhibit, No. AIAA-2011-5653, 2011.
} 
${ }^{6}$ Kopasakis, G., Connolly, J., and Seidel, J., "Propulsion System Dynamic Modeling of the NASA Supersonic Concept Vehicle for AeroPropulsoServoElasticity," 50th AIAA/ASME/SAE/ASEE Propulsion and Energy Forum, No. AIAA 2014-, 2014.

${ }^{7}$ Chavez, F. and Schmidt, D., "Analytical Aeropropulsive/Aeroelastic Hypersonic - Vehicle Model with Dynamic Analysis," Journal of Guidance, Control, and Dynamics, Vol. 17, No. 6, December 1994, pp. 1308-1319.

${ }^{8}$ Nguyen, N., "Integrated Flight Dynamic Modeling of Flexible Aircraft with Inertial Force-Propulsion-Aeroelastic Coupling," 44th AIAA Aerospace Sciences Meeting and Exhibit, No. AIAA 2008-194, Reno, Nevada, 7-10 January 2008.

${ }^{9}$ Clark, A., Wu, C., Mirmirani, M., and Choi, S., "Development of an Airframe-Propulsion Integrated Generic Hypersonic Vehicle Model," 46th AIAA Aerospace Sciences Meeting and Exhibit, No. AIAA 2006-218, Reno, Nevada, 9-12 January 2006.

${ }^{10}$ Biedron, R. T., Derlaga, J. M., Gnoffo, P. A., and et. al, "FUN3D Manual 12.4," Tech. Rep. TM-2014-218179, 2014.

${ }^{11}$ Anderson, J., "Computational Fluid Dynamics: The Basics with Applications," McGraw Hill, New York, NY, 1995.

${ }^{12}$ Chang, S.-C., "The Method of Space-Time Conservation Element and Solution Element - A New Approach for Solving the Navier-Stokes and Euler Equations," Tech. rep., NASA TM-111237, 1995.

${ }^{13}$ Connolly, J. and Kopasakis, G., "Loop Shaping Control Design for a Supersonic Propulsion System Model Using QFT Specifications and Bounds," 46th AIAA/ASME/SAE/ASEE Joint Propulsion Conference and Exhibit, No. AIAA-2010-7086, 2010.

${ }^{14}$ Lytle, J. K., "The Numerical Propulsion System Simulation: A Multidisciplinary Design System for Aerospace Vehicles," Tech. rep., NASA TM 1999-209194, 1999.

${ }^{15}$ Seldner, K., Mihaloew, J., and Blaha, R., "Generalized Simulation Technique for Turbojet Engine System Analysis," Tech. rep., NASA TN-D-6610, 1972.

${ }^{16}$ Kopasakis, G., "Feedback Control Systems Loop Shaping Design with Practical Considerations," Tech. rep., NASA TM 2007-215007, September 2007.

${ }^{17}$ Horowitz, I., "Quantitative Feedback Theory," IEEE Proceedings, Vol. 129, No. 6, Nov. 1982, pp. 215-226. 